Revue internationale de l'économie sociale

Recma

Créer et manager une association loi 1901 : une gestion au service de valeurs éthiques, Jérôme Boncler, Patrick Valéau. De Boeck, 2010, 234 pages

\title{
David Hiez
}

Numéro 319, janvier 2011

URI : https://id.erudit.org/iderudit/1020813ar

DOI : https://doi.org/10.7202/1020813ar

Aller au sommaire du numéro

\section{Éditeur(s)}

Association Recma

\section{ISSN}

1626-1682 (imprimé)

2261-2599 (numérique)

Découvrir la revue

Citer ce compte rendu

Hiez, D. (2011). Compte rendu de [Créer et manager une association loi 1901 : une gestion au service de valeurs éthiques, Jérôme Boncler, Patrick Valéau. De Boeck, 2010, 234 pages]. Revue internationale de l'économie sociale, (319),

118-120. https://doi.org/10.7202/1020813ar 
différentes formes d'argumentation pour développer leurs actions (créativité, convivialité, solidarité, engagement).

\section{Usages militants}

Commerce équitable, systèmes d'échanges locaux, responsabilité sociale del'entreprise, trois approches sectorielles des pratiques militantes étudiées autour du paradoxe de "l'entre-soi pour les autres ». L'étude du positionnement militant de ces organisations montre une forme d'usage politique qui permet la traduction de valeurs universelles dans un projet économique et social pragmatique. Cependant, l'observation de pratiques autocentrées portées par des communautés exclusives révèle une contradiction entre l'appartenance à un mode d'action et son impact plus global sur les territoires.

La conclusion de l'ouvrage souligne le lien historique entre l'invention de nouvelles formes de solidarités économiques et sociales et les crises qui affectent le modèle macroéconomique dominant. L'ESS apparaît ainsi à l'échelle des territoires comme une alternative crédible, ce qui justifie la mobilisation d'acteurs publics et privés engagés dans la mise en œuvre territoriale de nouvelles dynamiques produisant de nouveaux liens entre action publique et initiatives de la société civile.

Alain Penven,

Collège coopératif, Cercoop, Rennes-2

\section{Créer et manager une association loi 1901 : une gestion au service de valeurs éthiques Jérôme Boncler, Patrick Valéau. \\ De Boeck, 2010, 234 pages.}

Les éditions belges De Boecke, au sein d'une nouvelle collection " Petite entreprise et entrepreneuriat ", ont édité un nouveau titre, preuve du dynamisme croissant des éditeurs bruxellois sur le marché français: Créer et manager une association loi 1901. Rédigé par deux maitres de conférences en gestion français, le livre essaie de dépasser ce cadre strict, à moins qu'il ne soit la manifestation de la tendance expansionniste de cette nouvelle science sociale. Quoi qu'il en soit, le livre se démarque des guides classiques qui foisonnent sur les associations, qu'ils émanent de gestionnaires ou de juristes.

\section{Visées théoriques et pratiques}

L'intérêt de l'entreprise consiste dans le pari des auteurs: faire un ouvrage à dimension théorique, mais à visée pratique, avec certainement une prédominance du second aspect. Le public cible est en effet avant tout celui de dirigeants (actuels ou potentiels) d'association, pour les aider à comprendre les outils de gestion auxquels ils devront faire face et ainsi en tirer profit, au lieu de les vivre comme des pensums imposés par la réglementation ou des financeurs bureaucratiques. Cependant, ces outils ne sont pas présentés de façon didactique ou synthétique, ils sont plutôt exposés dans leur diversité et leurs fonctions, comme une occasion de pousser le lecteur à réfléchir sur la vie de son association et la manière dont elle appréhende les questions de gestion abordées. Pour stimuler la réflexion, le livre présente de nombreux documents de contextualisation très bienvenus: citations d'acteurs associatifs, divers dans leur statut et le type d'association dont ils émanent; comptes rendus de conseil d'administration ou d'assemblée générale.

La première partie de l'ouvrage tente d'identifier et de cerner ce que sont les associations, tant au regard de leurs valeurs (chapitre 1) qu'au regard de leur poids en France (chapitre 2). La deuxième partie adopte une perspective entrepreneuriale, en partant de l'émergence et de la conception du projet (chapitre 3) pour s'arrêter sur le principal moteur de cette dynamique, à savoir les entrepreneurs collectifs ou individuels (chapitre 4). La troisième partie étudie la conduite des associations, d'abord sous l'angle de leur direction ou, pour être plus mode, de leur gouvernance (chapitre 5), puis avec des objets plus techniques tels que la finance, la gestion des ressources humaines et le marketing (chapitre 6). Ne s'agissant pas d'une recherche fondamentale 
mais d'un outil, celui-ci ne comporte pas de conclusion explicitement développée. Pourtant, chaque chapitre s'achève par une conclusion et une application pratique, signe que le livre n'est pas qu'un exposé de règles techniques, mais qu'il fournit une ligne directrice, qu'il propose une lecture de la gestion des associations aujourd'hui.

\section{Accepter les tensions}

Et en effet, la plupart des chapitres font apparaître une tension dans la vie des associations, tension bien connue entre le bénévolat et le professionnalisme, le social et l'économique, le gratuit et l'entreprise sociale... Et ce n'est pas sous cet angle que le livre est le moins intéressant, bien au contraire. Alors que l'approche gestionnaire est traditionnellement perçue comme intrinsèquement liée à l'insertion au sein du monde associatif des considérations capitalistes, les deux auteurs montrent qu'il est parfaitement possible de faire un autre usage des concepts et outils des sciences de gestion. Dès le premier chapitre, en effet, les auteurs mettent en garde contre la tentation de laisser de côté les valeurs associatives traditionnelles au profit de l'efficacité et estiment d'ailleurs que cette tendance est déjà en recul. C'est le même discours tout au long des chapitres, montrant par exemple l'intérêt d'un business plan (chapitre 3) ou l'utilité d'une approche marketing (chapitre 6). Il s'agit d'une voie moyenne, dont on connaît les détracteurs habituels sur le thème d'un juste milieu qui veut concilier les inconciliables. Il y a toutefois là une démarche plus constructive et peut-être plus pragmatique: au lieu de rejeter l'appel à un mode de concevoir en vogue tant auprès des décideurs que des chercheurs, ce qui serait contre-productif, il s'agit d'intégrer dans le moule ainsi mobilisé les éléments spécifiques de l'objet analysé.

La limite de cette entreprise réside dans le risque de travestissement de la réalité associative par le simple usage de la logique gestionnaire. Pourtant, au fil de la lecture, je n'ai pu m'empêcher de voir dans cette logique gestionnaire des recettes de bon sens, depuis longtemps utilisées inconsciemment, ce que relève l'ouvrage lui-même, et que la gestion ne fait aujourd' hui que formaliser. Sous cet angle, parallèlement au fameux isomorphisme institutionnel par rapport aux entreprises capitalistes, les associations seraient soumises comme toutes les entreprises à un système de rationalisation et de systématisation, les deux mouvements ne pouvant se confondre.

\section{Les associations: des entreprises sociales?}

Compte tenu de l'actualité de la question, je relèverai la participation du livre au débat de l'entreprise sociale. Cette question a le mérite de montrer le respect que nos deux gestionnaires tâchent de promouvoir à l'égard de la spécificité associative. "Notre première objection à ce rapprochement entre les entrepreneurs lucratifs à dimension sociale et les entrepreneurs associatifs porte sur la nature de leurs objectifs. Les entrepreneurs sociaux parlent de buts économiques associés à des buts sociaux. Mais qu'entendent-ils par là? L'approche d'entreprise suit une double finalité, la finalité économique désignant la rentabilité et le profit, la finalité sociale concernant le bien-être des salariés, des usagers, voire de l'environnement. Pour les entrepreneurs associatifs le profit n'existe pas en tant que tel, pas en tant que fin, la finalité étant d'emblée sociale. Dans les associations, les rationalités technicoéconomiques se combinent aux rationalités en valeur. Notre seconde objection porte sur l'agencement de ces buts. Les hybridations de l'entrepreneuriat social ont, tout comme pour les associations, plusieurs buts. Mais les rapports entre ces derniers ne sont pas identiques suivant qu'il s'agisse d'une entreprise ou d'une association. Pour les entreprises, il s'agit le plus souvent d'optimiser le profit sous condition d'un minimum de respect pour l'environnement et les salariés. Pour les associations, le respect des valeurs peut devenir une fin en soi " (p. 124).

\section{Réflexions sur les pratiques}

C'est ce positionnement qui se retrouve, sur d'autres lignes de fracture, tout au long de l'ouvrage et qui lui attire la sympathie de ceux qui, comme moi (faute avouée est à moitié 
pardonnée), nourrissent quelques difficultés à mener, sans incompréhension, des discussions scientifiques avec les gestionnaires.

Pour finir, nous voudrions relever l'ambiguité fondamentale du livre. Comme nous l'avons dit, il n'est pas habituel: alors qu'il s'adresse à des acteurs de terrain, il ne leur fournit aucune recette ni même aucune explication technique détaillée sur la façon de monter un business plan ou une fiche de paie de directeur. En revanche, il propose des grilles de lecture de ces divers outils et invite sur cette base les acteurs à réfléchir à leur projet ou à leurs pratiques. Ceci est certainement beaucoup plus important et intelligent, tant il est vrai qu'aucun technicien ne pourra le faire à la place des acteurs associatifs eux-mêmes.

\section{David Hiez}

\section{Proudhon et la Banque du peuple Olivier Chaïbi. Connaissances et Savoirs, "Economie et gestion, histoire ", 2010, 230 pages.}

Les économistes et les sociologues, férus d'histoire de la banque et des banquiers en France, se réfèrent généralement aux travaux d'Hubert Bonin (1992). En effet, ils y trouvent à chaque fois non seulement un panorama détaillé du fonctionnement des institutions bancaires et des mécanismes financiers, mais aussi des éléments de compréhension du rôle et de l'image du banquier dans la société. L'ouvrage d'Olivier Chaïbi procède de cette veine, en se consacrant à ce projet malheureux de la Banque du peuple. Mais pourquoi s'intéresser à un ouvrage sur la Banque du peuple dans une revue d'économie sociale?

\section{Au service des ouvriers et des paysans}

Lauteur nous donne la réponse: « La Banque du peuple se présentait comme le centre d'associations qui devaient créer des caisses de secours mutuels, et fonctionner comme des coopératives de production et de consommation. Pour la première fois, un projet reliait les trois institutions sur lesquelles repose l'économie sociale » (p. 218). En outre, l'ambitieux projet de P.-J. Proudhon (1809-1865) consistait en un projet politique qui passe à la fois par "une réforme radicale du crédit et de la circulation monétaire " et par la recherche d'une réponse concrète aux besoins des paysans et des ouvriers. Cet état de fait est d'autant plus actuel que "crise et manque de confiance sont deux mots caractéristiques de l'état de la France en 1848 " (p. 13)! Trouver par l'économie bancaire une solution au problème social avait été tenté en avril 1849, à Metz et à Nancy, en liant le Mont-de-Piété, dont l'objet central est le crédit aux pauvres, et les Caisses d'épargne, dont l'objet est d'accueillir les dépôts des petits épargnants. Sans lendemain, cette idée faisant du " crédit " un outil de politique sociale s'imposa au point qu'elle fut reprise dans la Déclaration du peuple du 7 novembre 1848, en considérant que "le crédit privé, qui cause quand il est seul des désastres périodiques et d'incessantes iniquités, doit être modéré et complété par un vaste crédit social, non dans l'intérêt de quelques-uns, mais au bénéfice de tous $\%$. Bien que cette idée ne plaise pas aux économistes de l'époque, c'est-à-dire aux partisans de l'économie libérale, elle renforce la pertinence des expérimentations du microcrédit social depuis la fin des années 90 par les Crédits municipaux, en particulier.

\section{La voie d'un mutuellisme à retrouver}

Projet citoyen de lutte contre les inégalités, on pourrait regretter que la Banque du peuple n'ait pas opté pour un statut coopératif, lui préférant celui de société en nom collectif et en commandite. Mais, comme le rappelle à juste titre l'auteur, "la qualité d'actionnaire est accessible au petit peuple à raison de 5 francs sur dix mois, soit 50 centimes d'euro ou le prix d'une bouteille de vin " (p. 84). Au-delà de la responsabilisation solidaire attendue par P.-J. Proudhon, méfiant vis-à-vis des associations, l'article 6 des statuts de la Banque du peuple précise que " tout citoyen est appelé à faire partie de la Banque du peuple à titre de coopérateur. Il suffit pour cela d'adhérer 